\title{
Anti-Interference Ability Analysis for Common Atrial Fibrillation Features
}

\author{
Ying Li, Fan Zhou, Lina Zhao, Xiangyu Zhang, Jianqing Li and Chengyu Liu \\ School of Instrument Science and Engineering, Southeast University, Nanjing, China
}

\begin{abstract}
Recently, a number of RR interval rhythm-based atrial fibrillation $(A F)$ features have been developed and can achieve high classification accuracy for identifying $A F$ from normal rhythm ECGs on clean signals. However, in dynamic ECG monitoring scenario, accurate location for QRS complexes is challenging, resulting in a deviation between calculated and reference RR interval sequences. This deviation can cause the failure of $A F$ detection but its effect has not been quantified. This study addressed this concern and analyzed the anti-interference abilities of 14 commonly used AF features. Two types of Experiment were performed on the MIT-BIH AF database to simulate the deviation caused by QRS detection: 1) randomly moving forward or back several (0-15) labeled QRS locations to stimulate false detections, and 2) randomly missing several (0-9) labeled QRS locations to stimulate missing QRS detections. And the change in classification accuracy was regarded as a measure for evaluating the anti-interference ability of each feature. The results showed that features of AFEv, MAD, NFEn, COSEn and minRR showed high antiinterference abilities in both Experiments.
\end{abstract}

\section{Introduction}

Atrial fibrillation (AF) is the most common persistent arrhythmia, defined as 'tachyarrhythmia characterized by predominantly uncoordinated atrial activation with consequent deterioration of atrial mechanical function' [1]. The worldwide prevalence and incidence of AF are increasing year by year, and it has become a global epidemic and major public health problem [2]. However, current diagnose for AF is under-detected and underdiagnosed since AF can be non-specific or asymptomatic, thus can elude clinical detection. Therefore, using signal processing and machine learning combined with 24-hour dynamic electrocardiogram to detect $\mathrm{AF}$ signals has become a research hotspot.

There are two main types for AF analysis: atrial activity analysis-based and ventricular response analysis-based methods. The ventricular response analysis-based method is based on the predictability of RR interval to detect AF. It has become the mainstream in the field of AF detection due to its simple implementation and high accuracy, including coefficient of variation and density histogram [3],
Lorenz diagram [4][5], coefficient of sample entropy [6] and so on.

A large number of experiments showed that the result of a single feature on the MIT-BIH database was good, and combined with multi-features and machine learning methods, the result got better [7]. In fact, QRS detection will cause a deviation between the calculated and reference RR interval sequences. However, the RR interval sequence deviation has not been effectively quantified in current studies, and there is no analytical study on the antiinterference ability of AF features.

The aim of this study was to evaluate the influence of the RR interval sequence deviation caused by QRS detection to $\mathrm{AF}$ detection by means of adding interferences to RR interval sequences, thus finding out features with strong anti-interference ability by analyzing the changes in classification accuracy.

\section{Methods}

\subsection{Data}

MIT-BIH AF database is used in this study, which includes 25 long-term ECG records containing rhythm and beat annotation files. A single ECG recording lasts for 10 hours and is sampled at $250 \mathrm{~Hz}$. There are four types of rhythm annotations: AF (atrial fibrillation), AFL (atrial flutter), J (AV junction rhythm), and $\mathrm{N}$ (all other rhythms) [8].

In this study, AF and non-AF rhythms (N, J and AFL) were used for 2-type classification. Each rhythm was separated segment-by-segment, and each segment had 30beat in length. In order to test the anti-interference ability for each feature, we randomly selected 990 30-beat RR segments as training set and 330 30-beat RR segments as test set. The information of the selected datasets is shown in Table 1.

Table 1. Datasets using in this study.

\begin{tabular}{cccc}
\hline \multirow{2}{*}{ Dataset } & \multicolumn{3}{c}{ Rhythm type } \\
\cline { 2 - 4 } & AF & non-AF & Total \\
\hline Training & 660 & 330 & 990 \\
Test & 210 & 120 & 330 \\
\hline
\end{tabular}

\subsection{Features}


Feature extraction can represent the raw data in a lowdimension space. In this study, we selected 14 RR interval features proposed in earlier studies [9], which were listed as follows:

Time domain features: the mean value (mRR), minimum value (minRR), maximum value (maxRR), standard deviation (SDNN) of RR interval sequence, the square root of the mean squared differences of successive RR intervals (RMSSD), the median value of heart rate (medHR) and the percentage of RR intervals larger than $50 \mathrm{~ms}$ (PNN50).

Frequency domain features: normalized low frequency power (LFn), normalized high frequency power (HFn) and ratio of low frequency power to high frequency power (LF/HF).

Nonlinear features: coefficient of sample entropy (COSEn), coefficient of normalized fuzzy entropy (NFEn) $[10]$, the median of the variation in the absolute standard deviation from mean of heart rate in three adjacent RR segments (MAD) and AF evidence feature (AFEv).

\subsection{Experimental procedure}

The experiments aimed to find the features with strong anti-interference ability through simulating false or missing QRS detection, thus artificially adding interferences to the RR segments. The experimental procedure can be divided into four parts:

1) artificially adding interferences (false or missing QRS detection) to the RR segments. False QRS detection was set as Experiment A and missing QRS detection was set as Experiment B. Figure 1 shows the examples of these two cases.

2) calculating each feature on both original RR segments and RR segments with interferences.

3) using LIBSVM for classification. Since a single feature is one-dimensional, SVM is linearly inseparable in low-dimensional space, and the separating hyperplane is a point. The kernel function can map the original onedimensional feature space to the two-dimensional feature space, thus solving above question [11].

4) comparing the classification accuracies on both original RR segments and RR segments with interferences, and calculating the decrease in classification accuracy when the original RR segments were contaminated with interferences.

In this study, false detection was defined as the situation where the R-wave location was floating up and down due to errors in QRS detection resulting in the changes in RR interval sequences. False detection was simulated by randomly moving forward or back several (0-15) labeled QRS locations with different numbers of beats and different intensities of noise interference. Normally, according to the central limit theorem, we considered the noise interference to follow a normal distribution. The position where the interference was added was randomly

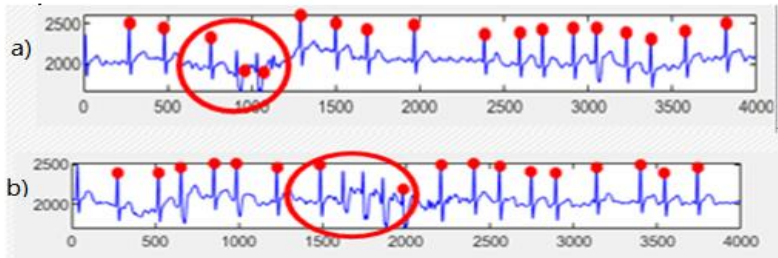

Figure 1. Example of a) false QRS detection and b) missing QRS detection.

generated. The intensity of noise interference was divided into two types: low intensity and high intensity. Lowintensity interference was defined as a normal distribution with a mean value of $40 \mathrm{~ms}$ and a standard deviation of 80 $\mathrm{ms}$, recorded as $N(40,80)$. High-intensity interference obeyed the normal distribution of $N(120,80)$. Figure 2 shows an example with 15 beats were changed. It can be seen that the RR sequence has a slight fluctuation after adding low-intensity interference, and its regularity changes little. After adding high-intensity interference, the RR sequence becomes disordered, and some features will falsely detect non-AF as AF.

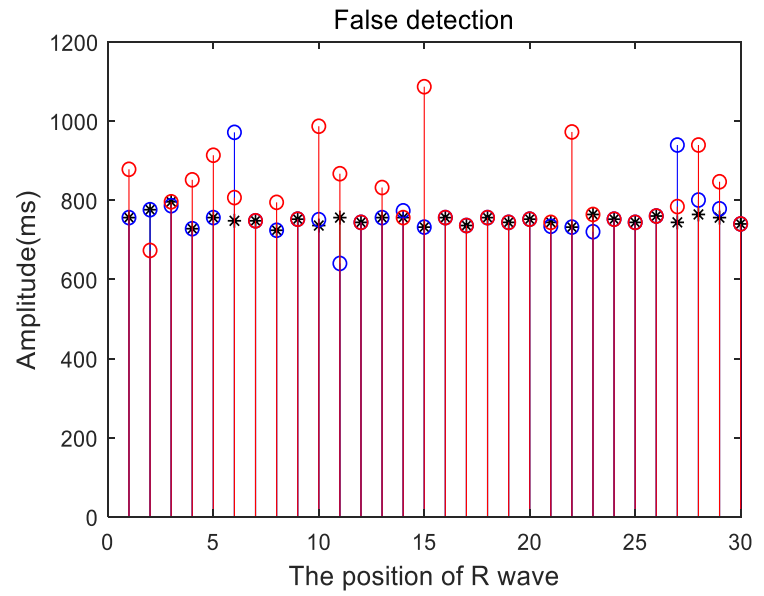

Figure 2. Comparison of the original RR sequence (marked in black) and sequences after adding low (blue) and high (red) intensity interferences.

Missing QRS detection was defined as the situation where some locations of QRS complexes were directly deleted. It was caused by the presence of noise or low ECG amplitude, inducing one or more adjacent RR intervals become longer, which would have a great impact on the AF detection. Missing QRS detection was simulated by randomly deleting several (0-9) labeled QRS locations. Figure 3 gives a comparison of RR sequence before and after 6-beat missing detection. Missing detection has a great damage to the regularity of RR sequence, so it is expected that the classification accuracy for each feature will decrease significantly in this case. 


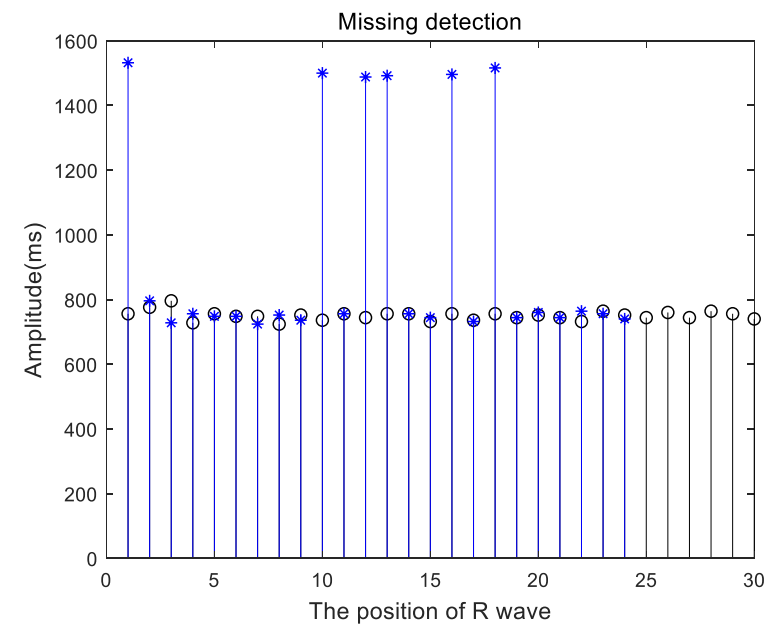

Figure 3. Comparison of the original RR sequence (black) and sequence after adding missing QRS detection (blue).

\section{Results and Discussions}

With the increase of interference, whatever the number of beat or the noise intensity, the classification accuracy is expected to decrease. The change in classification accuracy was regarded as a measure for evaluating the antiinterference ability. Features with good anti-interference ability can output slowly dropped and high-level accuracy. Figures 4 and 5 show the performance of each feature on the two situations: false detection and missing detection. Table 2 lists the accuracy results. Note that, the selected experimental data was only a small part of all MIT-BIH AF database, and these data have obvious $\mathrm{AF}$ or non- $\mathrm{AF}$ characteristic. Thus, the features reported high classification accuracy on the test data, and some features even report $100 \%$ classification for the selected original AF and non-AF signals.

Time-domain features of SDNN, RMSSD and PNN50 showed good anti-interference abilities in Experiment A but not in Experiment B. In Experiment B, they had high accuracy only in original RR segments. Once the missing detection appears, their classification accuracies decreased rapidly. That is because missing detection does a severer damage to the regularity of data than false detection. So the automatic analysis results of AF in the noisy situations should cause careful attention. Frequency-domain features of HFn, LFn, LF/HF, as well as time-domain features of $\mathrm{mRR}, \operatorname{maxRR}$ and medHR are thought to have very little anti-interference ability in both Experiments $\mathrm{A}$ and Experiments B. Their classification accuracies are relatively low and they prone to falsely detected lots of non-AF signals as AF. In addition, their accuracy curve varied irregular with the increase of interference intensity, reflecting they are inherently less suitable for identifying $\mathrm{AF}$ and non-AF signals.

In general, anti-interference ability of nonlinear features is better than the time-domain and frequency-
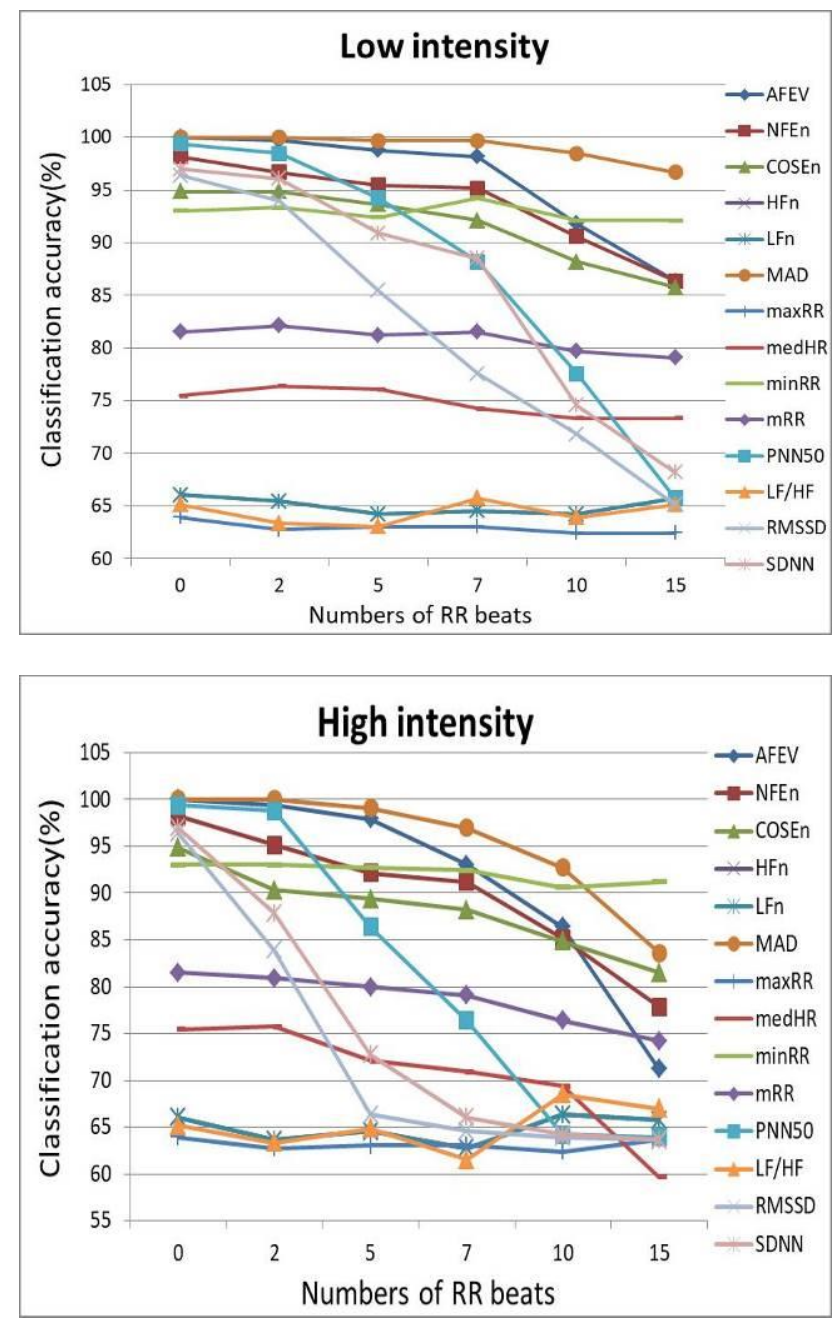

Figure 4. Results of anti-interference test of AF features on the Experiment of false QRS detection scenario.

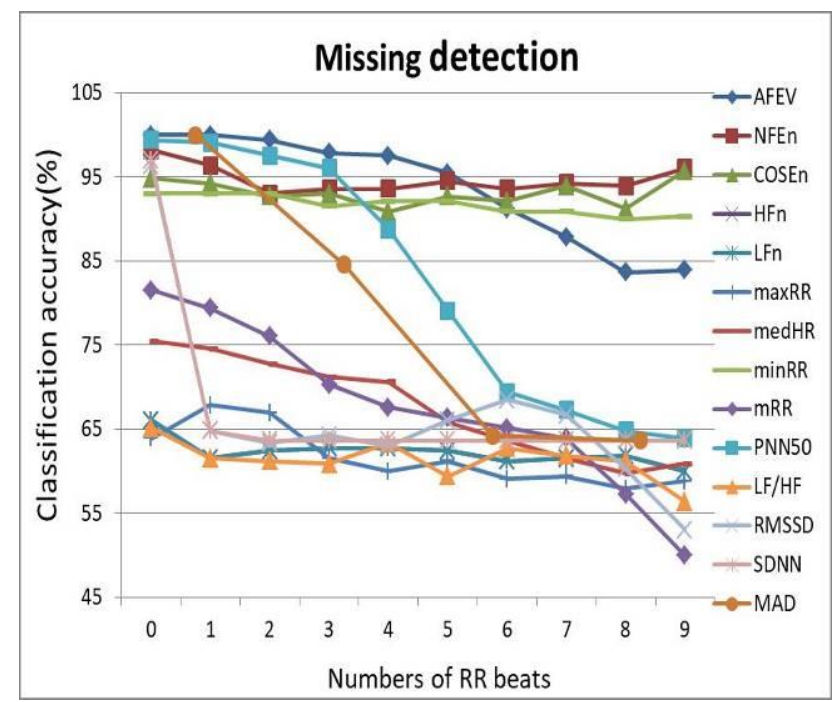

Figure 5. Results of anti-interference test of AF features on the Experiment of missing QRS detection scenario. 
Table 2. Results of each feature in test set.

\begin{tabular}{cccccccccc}
\hline \multirow{2}{*}{ Feature } & \multicolumn{4}{c}{ Accuracy $(\%)$} & \multirow{2}{*}{ Feature } & \multicolumn{4}{c}{ Accuracy $(\%)$} \\
\cline { 2 - 4 } \cline { 8 - 10 } & Original & A1 & A2 & B & & Original & A1 & A2 & B \\
\hline AFEv & 100.0 & 86.36 & 71.21 & 83.94 & medHR & 75.45 & 73.33 & 59.70 & 60.91 \\
NFEn & 98.18 & 86.36 & 77.88 & 96.06 & minRR & 93.03 & 92.12 & 91.21 & 90.30 \\
COSEn & 94.85 & 85.76 & 81.51 & 95.76 & mRR & 81.51 & 79.09 & 74.24 & 50.00 \\
HFn & 66.06 & 65.76 & 65.76 & 60.00 & PNN50 & 99.39 & 65.76 & 63.94 & 63.94 \\
LFn & 66.06 & 65.76 & 65.76 & 60.00 & LF/HF & 65.15 & 65.15 & 66.97 & 56.36 \\
MAD & 100.0 & 96.67 & 83.84 & 63.64 & RMSSD & 96.36 & 65.15 & 63.64 & 53.03 \\
maxRR & 63.94 & 62.42 & 63.64 & 58.79 & SDNN & 96.97 & 68.18 & 63.64 & 63.64 \\
\hline
\end{tabular}

A1: classification accuracy in low intensity test of experiment A; A2: classification accuracy in high intensity test of experiment A; B: classification accuracy in experiment B.

domain features. Features of AFEv, MAD, NFEn, COSEn and minRR always achieve high accuracy, which are thought to have strong anti-interference ability. The antiinterference ability of feature SDNN, RMSSD and PNN50 depends on the noise intensity of ECGs. However, features of HFn, LFn, LF/HF, mRR, maxRR and medHR always show bad anti-interference ability.

This study is only a pilot study and the in-depth analysis of anti-interference abilities of these $\mathrm{AF}$ features requires more experimental works.

\section{Conclusion}

This study simulated the deviation caused by QRS detection by adding interferences to the RR interval sequence in order to test the anti-interference ability of common AF feature. The results indicated that the features of AFEv, MAD, NFEn, COSEn and minRR are ideal features with strong anti-interference abilities.

\section{Acknowledgement}

The study was partly supported by the National Natural Science Foundation of China (81871444 and 61571113), the Natural Science Foundation of Jiangsu Province (BE2017735). The authors thank the support from the Southeast-Lenovo Wearable Heart-Sleep-Emotion Intelligent monitoring Lab.

\section{References}

[1] Fuster V, Rydén L E, Cannom D S, et al. ACC/AHA/ESC Guidelines for the Management of Patients With Atrial Fibrillation. Circulation, 2001, 104(17):2118-2168.

[2] Chugh S S, Havmoeller R, Narayanan K, et al. Worldwide Epidemiology of Atrial Fibrillation: A Global Burden of Disease 2010 Study. Circulation, 2014, 129(8): 837-847.

[3] Tateno K, Glass L. Automatic Detection of Atrial Fibrillation Using the Coefficient of Variation and Density
Histograms of $R R$ and $\Delta R R$ Intervals. Medical and Biological Engineering and Computing, 2001, 39(6): 664671.

[4] Sarkar S, Ritscher D, Mehra R. A Detector for a Chronic Implantable Atrial Tachyarrhythmia Monitor. IEEE Transactions on Biomedical Engineering, 2008, 55(3): 1219-1224.

[5] Ruan X, Liu C, Liu C, et al. Automatic Detection of Atrial Fibrillation Using RR Interval Signal. Proceedings of 4th International Conference on Biomedical Engineering and Informatics (BMEI 2011). IEEE, 2011, 2: 644-647.

[6] Lake D E, Moorman J R. Accurate Estimation of Entropy in Very Short Physiological Time Series: The Problem of Atrial Fibrillation Detection in Implanted Ventricular Devices. American Journal of Physiology-Heart and Circulatory Physiology, 2010, 300(1): H319-H325.

[7] Clifford G D, Liu C, Moody B, et al. AF Classification from a Short Single Lead ECG Recording: The PhysioNet/ Computing in Cardiology Challenge 2017. Computing in Cardiology 2017. IEEE, 2017, 44: 469(1)-469(4).

[8] Zhao L, Liu C, Wei S, et al. A New Entropy-Based Atrial Fibrillation Detection Method for Scanning Wearable ECG Recordings. Entropy, 2018, 20(12):904(1)-904(15).

[9] Li Q, Liu C, Oster J, et al. Signal Processing and Feature Selection Preprocessing for Classification in Noisy Healthcare Data. Machine Learning for Healthcare Technologies, 2016, 2: 33.

[10] Liu C, Oster J, Reinertsen E, et al. A Comparison of Entropy Approaches for AF Discrimination. Physiological Measurement, 2018, 39(7): 4002-4019.

[11] Chang C C, Lin C J. LIBSVM: A library for support vector machines. 2011.

Address for correspondence.

Jianqing Li and Chengyu Liu

School of Instrument Science and Engineering, Southeast University, China

E-mail: 1jq@seu.edu.cn and chengyu@ seu.edu.cn 